\title{
Towards Optimal Transmission Switching in Day-Ahead Unit Commitment
}

\author{
Kwok W. Cheung, Jun Wu \\ Alstom Grid, Inc., Redmond, Washington, USA \\ Email: kwok.cheung@alstom.com, jun.x.wu @alstom.com
}

Received December 2014

\begin{abstract}
Co-optimizing transmission topology with generation dispatch, and leveraging grid controllability could be a viable way to improve economic efficiency of system operations in control centers. In particular, day-ahead unit commitment is a typical business process that electric utilities deploy to ensure enough generation capacity is committed day-ahead to meet the load for the next day. In a market environment, day-ahead reliability unit commitment (DA-RUC) performs a simultaneous solution of minimizing the cost of commitment for resources to meet forecasted load, net scheduled interchange and operating reserve requirements using security constrained unit commitment (SCUC). The commitment may then be further checked by running security constrained economic dispatch (SCED) to verify that the commitment solution can be feasibly dispatched subject to system constraints and activated transmission constraints for each hour identified in the study period. This paper applies Optimal Transmission Switching (OTS) in DA-RUC using some heuristic pre-screening techniques to reduce the dimension of the OTS problem. Various pre-screening methods of candidate transmission lines for switching are proposed and compared. Simulation results will be presented to demonstrate generation dispatch combined with OTS in each hour could reduce congestion cost and significantly lower generation cost.
\end{abstract}

\section{Keywords}

Transmission Switching, Market Efficiency, System Operation, Economic Dispatch

\section{Introduction}

The restructured electric power industry has brought new challenges for the secured and efficient operation of stressed power systems. In North America, almost all Regional Transmission Organizations (RTO) such as PJM, Midcontinent ISO or ISO New England, are fundamentally reliant on wholesale market mechanism to optimally dispatch energy and ancillary services of generation resources reliably over large geographical regions [1] [4]. A typical market system in North America supports a series of business functions for market and system operations. Depending on operational needs, the system can be configured to include one or more of the following business processes (Figure 1).

Reliability Unit Commitment (RUC) process provides system operators a set of tools to revise the day-ahead unit commitment schedule as necessary in order to ensure that the forecasted load and operating reserve 


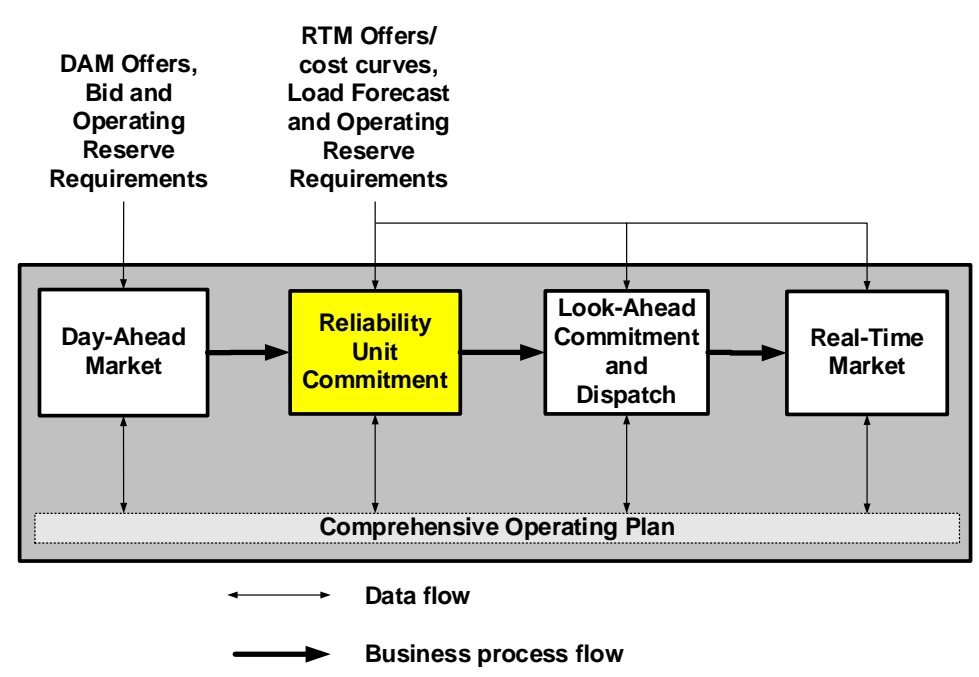

Figure 1. Business processes of a market system.

requirements will be met and the transmission system is reliable and secured. The RUC performs a co-optimized simultaneous solution to minimize the cost of commitment for resources to meet forecasted load, net scheduled interchange and operating reserve requirements using SCUC. The commitment may then be further confirmed by running SCED to verify that the commitment solution can be feasibly dispatched subject to system constraints and activated transmission constraints for each hour in the study period.

In recent years, energy systems whether in developed or emerging economies are undergoing changes due to the challenges imposed by smart grid. Optimal transmission switching [2] [3] seems to be a viable way to leverage grid controllability for enhancing system performance. Transmission control has been identified as a valuable mechanism for a variety of benefits, from improving the system reliability, stability to improving the market surplus and efficiency of the grid.

It is obvious that transmission systems could become smarter and more efficient when the control of network topology can be factored into the overall dispatch process of generation and transmission resources taking both system reliability and economics into consideration in a systematic fashion. The problem of OTS can be formulated as a mixed-integer programming problem. However, it requires the introduction of a significant number of integer variables for transmission line in/out statuses which becomes a key impediment of applying a full OTS scheme in any large power network. Fortunately, a practical solution to improve operational efficiency could be achieved by limiting the set of transmission line candidates for OTS.

The rest of the paper will be presented as follows. Section 3 addresses the basic model of OTS. Section 4 proposes selection methods of candidate transmission lines for a study of OTS in DA-RUC. Section 5 presents numerical results for the DA-RUC/SCED using OTS for a large-scale power system with more than 37,000 buses. Conclusions are in Section 6.

\section{0TS Modeling}

The core application of the market system is basically a centralized market clearing engine solving a unit commitment, scheduling and dispatch problem. The problem itself is typically formulated as a Mixed Integer Programming (MIP) problem [5]. A compact of form of such problem can be described as follows:

$$
\min \sum_{g, t}\left(u_{g t} \chi_{g t}\left(p_{g t}\right)+\varsigma_{g t}\left(u_{g(t-1)}, u_{g t}\right)\right)
$$

Subject to

$$
\begin{gathered}
\left(\lambda_{t}\right) \sum_{g} p_{g t}=l_{t}+p_{t}^{\text {loss }}, \quad \forall t \\
\left(\alpha_{t} \geq 0\right) \quad \sum_{g} r_{g t} \geq \underline{r}_{t}, \quad \forall t
\end{gathered}
$$




$$
\begin{gathered}
u_{g t} \underline{p}_{g t} \leq p_{g t} \leq u_{g t} \bar{p}_{g t}, \quad \forall g, t \\
p_{g t}+r_{g t} \leq u_{g t} \bar{p}_{g t}, \quad \forall g, t \\
0 \leq r_{g t} \leq u_{g t} \bar{r}_{g t}, \quad \forall g, t \\
\left(\mu_{k t}\right) \quad \underline{f k t}_{k t} z_{k t} \leq f_{k t} \leq \bar{f}_{k t} z_{k t}, \quad \forall k, t \\
B_{m n}\left(\theta_{m t}-\theta_{n t}\right)-f_{k t}+\left(1-z_{k t}\right) M_{k} \geq 0, \quad \forall k, t \\
B_{m n}\left(\theta_{m t}-\theta_{n t}\right)-f_{k t}-\left(1-z_{k t}\right) M_{k} \leq 0, \quad \forall k, t \\
\sum_{k}\left(1-z_{k t}\right) \leq j_{-} \text {Open, } \quad \forall k, t \\
\underline{\theta} \leq \theta_{m t} \leq \bar{\theta}, \quad \forall m, t \\
\left(\lambda_{m t}\right) \quad p_{g m t}-l_{m t}-p_{m t}^{\text {loss }}=\sum_{k \in \operatorname{line}_{m}^{f r}} f_{k t}-\sum_{k \in \operatorname{line}_{m}^{\mathrm{to}}} f_{k t}, \quad \forall m, t \\
\left|p_{g t}-p_{g(t-1)}\right| \leq u_{g t} R_{g t}, \quad \forall g, t \\
(u, p, r) \in \Gamma
\end{gathered}
$$

where $m$ and $n$ are "from" and "to" bus of transmission line $k$, respectively, and $z_{k t}$ listed in (7)-(10) is the binary variable representing the state of the transmission element; a value of 1 reflects a closed status and a value of 0 reflects an open status. $M_{k}$ listed in (8) and (9) is an arbitrarily large number. When the binary variable $z_{k t}$ is 1 , the value of $M_{k}$ is irrelevant. When the binary variable $z_{k t}$ is 0 , the value of $M_{k}$ should be big enough to ensure that (8) and (9) are satisfied regardless of the difference in the bus angles. For all practical purposes $M_{k}$ can be set to $B_{m n}(\bar{\theta}-\underline{\theta})$.j_Open listed in (10) denotes the maximum number of open lines.

The objective (1) is to minimize the production plus start-up costs. The minimization is subject to many constraints including supply and demand constraints (2), capacity constraints (4), (5), (6), ramp constraints (13), transmission constraints with transmission line switching (7), (8), (9), (10), (11), bus node power balance (12) and reserve requirements (3). It is important to note that the transmission flow can also be expressed as a linear function of bus net injections in the pre-contingent state.

$$
f_{k t}=\sum_{g} a_{k g t} p_{g t}, \quad \forall k, t
$$

Most RTO's real-time operation requires the $n-1$ security. Hence, the transmission flow presented here can either be pre-contingent or post-contingent line or interface flow. Other constraints, such as minimum up and down times constraints, ramp constraints, and operating and regulating etc. reserve requirements are considered as a part of (14).

After the unit commitment problem is solved, the integer variables $u_{g t}$ will be frozen, the optimal solution for the scheduling and dispatch problem will be reduced to a Linear Programming (LP) problem in which market clearing quantities and market clearing prices by location can be determined. The market clearing prices by location, called locational marginal prices are by-products of the optimization solution.

Locational marginal prices for energy can be written as:

$$
\mathrm{LMP}_{g t}=\lambda_{t}-\lambda_{t} \frac{\partial p_{t}^{\text {loss }}}{\partial p_{g t}}-\sum_{k} a_{k g t} \mu_{k t}
$$

The three terms in the above LMP equation could be interpreted as the three components of LMP namely energy, loss and congestion, respectively. These locational price results give precise representation of the cause-effect relationship that is consistent with grid reliability management.

\section{Transmission Line Switching Selection}

With the introduction of $z_{k t}$ in the OTS model, transmission switching adds substantial computational com- 
plexity to the market clearing engine. To be able to solve the MIP model for large scale systems, transmission lines are divided into two sets. One is a set of switchable transmission lines which are associated with decision integer variables as in (7)-(10). The other is a set of transmission lines which status (on/off) is predetermined before running optimization of market clearing.

The proper size of switchable transmission lines and the value of $j \_$Open in (10) are critical parameters that affect solution time. These parameters might vary from networks to networks and cases by cases. [6] has shown that solving OTS could be done within the required time in large electricity market systems by selecting a proper size of candidate transmission lines. However, it remains as a challenge on how to select a proper set of candidate transmission lines to get better objective savings among thousands of transmission lines for a given largescale power system. With a given limit of switchable transmission lines, the following criteria are proposed to be used for the selection of switchable transmission lines.

\subsection{Selection Criteria}

\subsubsection{Limit Violations of Transmission Lines}

For a given base case, if transmission lines violated their limits, the violation penalties will appear in the cost of the objective function. Switching off the violated transmission lines that does not cause any new violations will eliminate the high penalty costs introduced by those violated transmission lines. Hence, the violated transmission lines could be good candidates of switchable transmission lines.

\subsubsection{Binding Transmission Lines}

For a given base case, binding transmission lines are the resources to cause congestion and lead to dispatching more expensive generation. Switching off binding transmission lines that does not cause other lines to be congested could reduce congestion cost. Hence, binding transmission lines could also be good candidates of switchable transmission lines.

\subsubsection{Congestion Rents of Transmission Lines}

Congestion rent $(C R)$ [7] [9], which is defined as the difference in shadow prices between "from" and "to" side bus of a transmission line $k$, multiplied by the flow on the line.

$$
\mathrm{CR}=f_{k t}\left(\lambda_{m t}-\lambda_{n t}\right)
$$

By introducing a fictitious variable of "fraction of line out of service” [8] suggested to use (17) to rank transmission lines for switching. Due to complementary slackness, the shadow price of a transmission line is zero if there are no transmission constraints binding; negative if there are transmission constraints binding at upper limits; positive if there are transmission constraints binding at lower limits. The lines are congested when there are shadow price differences between "from" and "to" side of the line of interest. Switching off these transmission lines would possibly reduce the congestion costs.

\subsubsection{Production Costs Associated with Transmission Lines}

The production cost (PC) could be increased due to transmission congestion. In those cases, some higher-cost generation is dispatched in favor of lower-cost generation that would otherwise be used in the absence of transmission constraints.

In our previous research work [10], a formulation of the sensitivities of bus angle with respect to the flow of transmission line is developed:

$$
\frac{\partial \theta_{i t}}{\partial f_{k t}}=\beta_{i k}=B_{m n}\left(\Pi_{m i}^{-1}-\Pi_{n i}^{-1}\right)
$$

where elements of matrix $\Pi$ :

$$
\Pi_{i i}=\sum_{j \neq i} B_{i j}^{2}, \quad \Pi_{i j}=-B_{i j}^{2}
$$

The production cost associated with the transmission line $k$ is

$$
\mathrm{PC}_{k}=\left(\sum_{g} c_{g t}\left(B_{h h} \beta_{h k}-\sum_{i \neq h} B_{h i} \beta_{i k}\right)\right) f_{k}
$$


where $c_{g t}$ denotes the energy offer price of generator $g$ at time $t$ and $h$ denotes the bus to which generation $g$ is connected.

Intuitively, if the transmission line $k$ is congested, switching it off will cause $\mathrm{PC}_{k}$ to go to 0 . If switching off line $k$ does not cause more congestion on other lines, it will reduce the overall congestion cost. Thus PC associated with transmission line can be used as another selection criterion for transmission switching candidates.

\subsection{Selection Methods of Switchable Lines}

The purpose of selecting candidate transmission lines for switching is to identify those transmission lines which could eliminate or reduce congestion cost for a given power network. For all practical purposes, the OTS problem could be solved by selecting one candidate line at a time or limiting the maximum number of open lines. In either case, a proper size of candidate set of transmission lines [6] is crucial to make the problem tractable.

Table 1 illustrates OTS solver solution time for different size of candidate transmission lines (OTSbr) and different maximum number of open lines $j_{-}$Open. From Table 1, a practical number of switchable transmission lines are probably less than 20 and the maximum number of open lines $j_{-}$Open is about 10 . Tractable values of OTSbr and $j_{-}$Open could vary with the size of power network. However, these parameters usually do not vary much for a given power network and can be determined via offline studies.

The violated transmission lines, if any, will first be selected as switching candidates since they usually cause large penalty costs in the objective function. However, this criterion is usually not satisfied under normal system conditions. Instead, binding transmission constraints are more likely to be observed. Both CR and PC can be used as ranking measures for effective transmission switching candidates. However, combining PC and CR (PCCR) [10] could more effectively reduce overall objective cost.

It is important to note that neither CR, PCCR, nor binding transmission lines by itself is a perfect measure since they are all topology-dependent. In other words, there is no guarantee that new congestion will not occur when topology changes. Therefore, in the RUC/SCED OTS study, we propose to use the following methods to select candidate transmission lines:

1) OTS_CR—Rank transmission lines by congestion rent (CR), and select the higher ranking transmission lines as candidate transmission lines.

2) OTS_PCCR - Filter out transmission lines with non-positive $\mathrm{PC}_{k}$, rank filtered transmission lines by CR, and select the higher ranking transmission lines as candidate transmission lines.

3) OTS_binding_CR—Rank binding transmission lines by CR and select the higher ranking transmission lines that are binding as candidate transmission lines before selecting the non-binding ones.

4) OTS_binding_PCCR—Rank binding transmission lines by PCCR and select the higher ranking transmission lines that are binding as candidate transmission lines before selecting the non-binding ones.

\section{OTS Simulation Results}

We applied OTS to the DA-RUC process for one of the largest market systems in North America with more than 37,000 buses and 48,000 transmission lines.

- DA-RUC Case and SCED Objective Cost w/o OTS

The hourly load forecast, the number of binding transmission constraints and the objective costs of a $24 \mathrm{hr}$ DA-RUC case as a baseline solution (without OTS) are shown in Table 2. Hour 4 has the lowest load, hours 12 to 21 are the high load period. Hour 4 has the least number of binding transmission constraints equal to 16 and

Table 1. Solution time varies with OTSbr and j_Open.

\begin{tabular}{cccc}
\hline OTSbr & j_Open & Open Lines & Solution Time (sec) \\
\hline 14 & 10 & 1 & 351 \\
18 & 8 & 4 & 378 \\
18 & 18 & 4 & 378 \\
27 & 5 & 4 & 516 \\
74 & 5 & 5 & 920 \\
340 & 5 & 1 & 2444 \\
\hline
\end{tabular}


Table 2. Hourly load forecast, number of binding constraints \& objective cost for a DA RUC study case.

\begin{tabular}{|c|c|c|c|}
\hline Hour (h) & Load (Mw) & Number of Binding Constraints & Objective (\$/h) \\
\hline 1 & 48,640 & 20 & 847453.2 \\
\hline 2 & 46,577 & 20 & 810258.7 \\
\hline 3 & 45358.3 & 18 & 784169.9 \\
\hline 4 & 44837.9 & 16 & 776758.6 \\
\hline 5 & 45217.2 & 18 & 786803.7 \\
\hline 6 & 46350.2 & 17 & 808562.2 \\
\hline 7 & 47854.9 & 20 & 843179.1 \\
\hline 8 & 50048.8 & 24 & 907384.6 \\
\hline 9 & 53053.4 & 24 & 1009595.5 \\
\hline 10 & 55290.6 & 22 & 1200858.8 \\
\hline 11 & 56437.1 & 24 & 1213839.6 \\
\hline 12 & 56955.7 & 23 & 1292906.6 \\
\hline 13 & 57224.9 & 22 & 1262689.1 \\
\hline 14 & 57283.8 & 24 & 1190398.7 \\
\hline 15 & 57,213 & 24 & 1198609.9 \\
\hline 16 & 57356.2 & 28 & 1205419.0 \\
\hline 17 & 57477.8 & 26 & 1222535.2 \\
\hline 18 & 57268.2 & 27 & 1213888.9 \\
\hline 19 & 56610.3 & 26 & 1192487.8 \\
\hline 20 & 57332.1 & 29 & 1260312.3 \\
\hline 21 & 57213.5 & 28 & 1286752.8 \\
\hline 22 & 54646.3 & 25 & 1178705.5 \\
\hline 23 & 51117.7 & 21 & 1080567.1 \\
\hline 24 & 47,814 & 21 & 994112.2 \\
\hline
\end{tabular}

hour 20 has the highest number of binding transmission constraints equal to 29. The total objective cost for the whole day is $\$ 25,568,249$.

- DA RUC SCED OTS Results and Analysis

A group candidate transmission lines with a limit of 10 switchable candidate transmission lines is selected for each proposed method. Their simulation results of OTS are presented in Table 3.

Figure 2 depicts hourly objective costs for the proposed methods. In general, the objective savings during the peak hours starting from hour 9 are more than that in the valley hours between hours 1 to 8 .

Comparing to the baseline solution (w/o OTS), it is interesting to note that OTS objective costs are reduced for all hours except for hour 12 of OTS_binding_CR. Table 4 illustrates the total 24 hour objective cost for each method. OTS_PCCR achieves on average more objective savings in most hours compared to the rest of the other methods.

\section{Conclusions}

Optimal transmission switching can leverage grid controllability for enhancing system performance. Due to the scale of the problem, a limited set of transmission switching candidates are pre-selected for switching by an co-optimization algorithm of energy and network topology for all practical purposes. First, simulation results from a large network in the DA- RUC demonstrate that OTS provides more savings during the peak hours of a 
Table 3. Comparison of objective costs.

\begin{tabular}{|c|c|c|c|c|c|}
\hline \multirow{2}{*}{$\mathrm{Hr}$} & \multicolumn{3}{|r|}{ Objective } & \multicolumn{2}{|l|}{$(\$ / \mathbf{h})$} \\
\hline & w/o OTS & OTS-CR & OTS-PCCR & OTS_binding PCCR & OTS_binding CR \\
\hline 1 & 847,453 & 845,754 & 844,072 & 844,913 & 845,297 \\
\hline 2 & 810,259 & 806,667 & 805,545 & 806,869 & 809,141 \\
\hline 3 & 784,170 & 780,201 & 779,196 & 780,862 & 783,403 \\
\hline 4 & 776,759 & 772,239 & 771,687 & 772,567 & 776,207 \\
\hline 5 & 786,804 & 781,886 & 781,215 & 782,799 & 786,222 \\
\hline 6 & 808,562 & 803,937 & 803,922 & 804,449 & 807,356 \\
\hline 7 & 843,179 & 838,381 & 838,658 & 837,788 & 842,179 \\
\hline 8 & 907,385 & 889,834 & 890,768 & 904,569 & 906,945 \\
\hline 9 & $1,009,596$ & 991,478 & 991,434 & 971,654 & $1,004,996$ \\
\hline 10 & $1,200,859$ & 1,093,192 & $1,096,146$ & $1,113,900$ & $1,192,598$ \\
\hline 11 & $1,213,840$ & 1,115,292 & $1,098,780$ & $1,205,136$ & $1,210,633$ \\
\hline 12 & $1,292,907$ & $1,240,486$ & $1,143,344$ & $1,262,159$ & $1,292,906$ \\
\hline 13 & $1,262,689$ & $1,230,259$ & $1,138,566$ & $1,232,974$ & $1,250,874$ \\
\hline 14 & $1,190,399$ & $1,109,584$ & $1,112,472$ & $1,166,548$ & $1,181,088$ \\
\hline 15 & $1,198,610$ & $1,141,618$ & $1,121,518$ & $1,170,307$ & $1,192,757$ \\
\hline 16 & $1,205,419$ & $1,164,641$ & $1,141,122$ & $1,174,303$ & $1,189,568$ \\
\hline 17 & $1,222,535$ & $1,128,318$ & $1,160,128$ & $1,196,153$ & $1,202,415$ \\
\hline 18 & 1,213,889 & $1,205,853$ & $1,205,983$ & 1,098,233 & $1,210,899$ \\
\hline 19 & $1,192,488$ & $1,136,221$ & $1,138,401$ & $1,176,815$ & $1,189,683$ \\
\hline 20 & $1,260,312$ & $1,175,088$ & 1,173,320 & $1,235,244$ & $1,252,684$ \\
\hline 21 & $1,286,753$ & 1,151,641 & $1,142,653$ & $1,282,454$ & $1,284,152$ \\
\hline 22 & $1,178,705$ & 1,093,961 & $1,092,866$ & $1,170,503$ & $1,172,846$ \\
\hline 23 & $1,080,567$ & 923,572 & $1,003,549$ & $1,052,823$ & $1,076,384$ \\
\hline 24 & 994,112 & 841,332 & 852,076 & 960,907 & 989,619 \\
\hline
\end{tabular}

Table 4. Total 24-hour objective cost comparison.

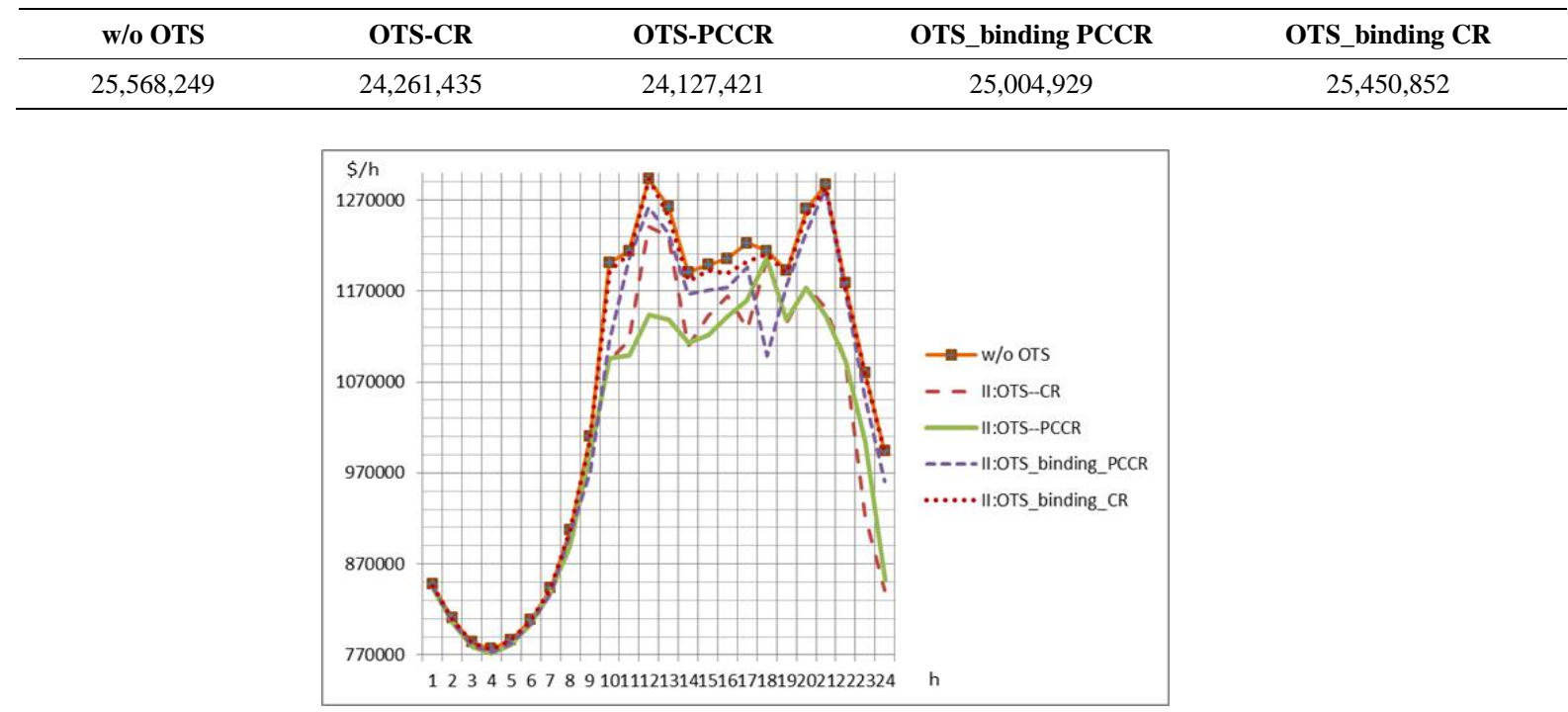

Figure 2. Hourly objective cost comparison. 
reasonably stressed system. Secondly, a smaller size of candidate transmission line requires less computation time but can still provide considerable objective savings. PCCR is likely a better selection criterion that of $C R$ in terms of objective savings. In some cases, applying PCCR to only the binding transmission lines could be a reasonable compromise if computation time is a concern.

\section{References}

[1] Cheung, K.W. (2008) Ancillary Service Market Design and Implementation in North America: From Theory to Practice. Panel Paper, Proceedings of the 3rd International Conference on Electric Utility Deregulation, Restructuring and Power Technologies (DRPT), Nanjuing, 6-9 April 2008, 66-73. http://dx.doi.org/10.1109/DRPT.2008.4523381

[2] Wood, A.J. and Wollenberg, B.F. (1996) Power Generation, Operation, and Control. 2nd Edition, John Wiley \& Sons, New York.

[3] Fisher, E.B., O’Neill, R.P. and Ferris, M.C. (2008) Optimal Transmission Switching. IEEE Transactions on Power Systems, 23, 1343-1355. http://dx.doi.org/10.1109/TPWRS.2008.922256

[4] Chow, J.H., deMello, R. and Cheung, K.W. (2005) Electricity Market Design: An Integrated Approach to Reliability Assurance. Invited Paper, IEEE Proceeding (Special Issue on Power Technology \& Policy: Forty Years after the 1965 Blackout), 93, 1956-1969.

[5] Cheung, K.W. (2011) Economic Evaluation of Transmission Outages and Switching for Market and System Operations. Panel Paper, Proceedings of 2011 IEEE PES General Meeting.

[6] Cheung, K.W., Wu, J. and Rios-Zalapa, R. (2010) A Practical Implementation of Optimal Transmission Switching. Proceedings of the 4th International Conference on Electric Utility Deregulation, Restructuring and Power Technologies (DRPT 2010).

[7] Hedman, K.W., O’Neill, R.P., Fisher, E.B. and Oren, S.S. (2008) Optimal Transmission Switching-Sensitivity Analysis and Extensions. IEEE Transactions on Power Systems, 23, 1469-1479. http://dx.doi.org/10.1109/TPWRS.2008.926411

[8] Fuller, J.D., Ramasra, R. and Cha, A. (2012) Fast Heuristics for Transmission Line Switching. IEEE Transactions on Power Systems, 27, 1377-1386. http://dx.doi.org/10.1109/TPWRS.2012.2186155

[9] Ruiz, P.A., Foster, J.M., Rudkevich, A. and Caramanis, M.C. (2011) On Fast Transmission Topology Control Heuristics. Proceedings of 2011 IEEE PES General Meeting.

[10] Wu, J. and Cheung, K.W. (2013) On Selection of Transmission Line Candidates for Optimal Transmission Switching in Large Power Networks. Proceedings of IEEE PES General Meeting, 21-25 July 2013, Vancouver, BC. 


\section{Nomenclature}

$g:$ Index for generator,

$t$ : Index for study period,

$k:$ Index for transmission line,

$m, n$ : Index for "from", "to" bus of transmission line $k$,

line $_{m}^{f r}$, line ${ }_{m}^{\text {to }}$ : Set of transmission lines whose "from", "to" sides are connected to bus $m$,

$l_{t}, l_{m t}$ : Load forecast for system and bus $\mathrm{m}$ for study period $t$,

$p_{g t}, \bar{p}_{g t}$ : Capacity limits of generator $g$ for study period $t$,

$\bar{r}_{+}$: Reserve requirement for study period $t$,

$\bar{r}_{g t}$ : Reserve limit of generator $g$ for study period $t$,

$R_{g t}$ : Ramp limit of generator $g$ for study period $t$,

$B$ : Susceptance of transmission line,

$\bar{f}_{k t}$ : Limit of transmission line $k$ for study period $t$,

$a_{k g t}$ : Sensitivity of transmission line $k$ with respect to generator $g$ for study period $t$,

$\theta_{m t}$ : bus angle of bus $m$ for study period $t$,

$\bar{\theta}, \underline{\theta}$ : High and low limits of bus angle for study period $t$,

$u_{g t}$ : Commitment status for generator $g$ for study period $t$;

$p_{g t}$ : Power output of generator $g$ for study period $t$,

$r_{g t}$ : Reserve contribution of generator $g$ for study period $t$,

$f_{k t}$ : Transmission line flow for study period $t$,

$\mathbf{u}$ : vector of all $u_{g t}, \mathbf{p}$ : vector of all $p_{g t}$,

$\mathbf{r}$ : vector of all $r_{g t}$,

$\Gamma$ : set of feasible solutions,

$\chi_{g t}(\cdot)$ : Production and no-load costs for generator $g$ for study period $t$,

$\varsigma_{q t}(\cdot)$ : Start-up costs for generator $g$ for study period $t$,

$p_{t}^{\text {loss }}(\cdot), p_{m t}^{\text {loss }}(\cdot)$ : Loss for system and bus $m$ for study period $t$. 